\title{
Distributed Ledgers in der Energieversorgung
}

\section{Technologien und Geschäftsmodelle}

\author{
Pascal Pfister und Jan T. Frecè
}

\section{Zusammenfassung}

Distributed Ledgers werden seit ihrer Entstehung 2009 von vielen Seiten als Mittel zur grundlegenden Umgestaltung ganzer Branchen angepriesen. Während die Technologie sicherlich Potenzial bietet, diverse Steuerungs- und Abwicklungsprozesse zu revolutionieren, bedingt eine solche Umstellung einerseits fundiertes Wissen hinsichtlich der Rollen und Prozesse im entsprechenden Wirtschaftsumfeld sowie der technologischen Grundlagen von Distributed Ledgers, um dauerhaft Mehrwert zu schaffen. Dieses Buchkapitel zeigt am Beispiel der Energieversorgung auf, welche Faktoren für eine mehrwertgenerierende, sichere Implementierung der Ledger-Technologie berücksichtigt werden müssen: von technologischen Fragen bis zur Frage nach neuen Geschäftsmodellen und den damit verbundenen Risiken.

\subsection{Einleitung}

Mit der stark zunehmenden Dezentralisierung der Energieproduktion und der immer intelligenter werdenden Stromnetze wird sich die Energiebranche in Zukunft in einem komplexen und sich schnell wandelnden Umfeld bewegen. Überdies soll der Strommarkt mit der Inkraftsetzung des neuen StromVG vollständig liberalisiert werden. Hier verspricht die Distributed Ledger Technology (DLT) respektive die Blockchain vielversprechende Lö-

P. Pfister

Bern, Schweiz

J. T. Frecè $(\bowtie)$

Berner Fachhochschule Wirtschaft, Bern, Schweiz

E-Mail: jan.frece@bfh.ch

(C) Der/die Autor(en) 2021 
sungen für unterschiedliche Geschäftsmodelle und Anwendungsfälle. Auf der Basis von diversen durchgeführten Studien, möchte dieser Beitrag einerseits einen möglichen neuen Anwendungsfall für den Verteilnetzbetreiber (VNB) untersuchen und andererseits den aktuellen Boom, um die Technologie in der Energiewirtschaft kritisch hinterfragen. Des Weiteren spielen die regulatorischen Rahmenbedingungen eine zentrale Rolle, weshalb die dadurch entstehenden Grenzen ebenfalls aufgezeigt werden sollen. Der Anwendungsfall respektive die Anforderungen an eine Blockchain beziehen sich ausschließlich auf den Verteilnetzbetreiber beziehungsweise auf die Abwicklung der Netznutzungsentgelte und auf das Fördern von netzdienlichem Verhalten und lässt andere Aspekte wie z. B. Endkundendienstleistungen außen vor. Hierzu wird ein mögliches Geschäfts- und Anwendungsfeld der Blockchain für den VNB beschrieben und dafür folgenden Fragen beantwortet:

- Welchen Mehrwert und welche Risiken bringt die Blockchain für den VNB?

- Welche neuen Geschäftsmodelle oder Anwendungsfälle der Blockchain könnten sich in der Zukunft für den VNB eröffnen?

- Welche Vor- und Nachteile bringen diese Modelle mit sich?

\subsection{Ausgangslage}

Mit der Energiestrategie 2050 hat sich die Schweizer Bevölkerung für eine dezentrale Stromproduktion und für die Förderung von erneuerbarer Energie entschieden. Dies hat zu mehreren Anpassungen im StromVG geführt. Der Bundesrat hat am 17. Oktober 2018 die Vernehmlassung der Vorlage in Auftrag gegeben, welche vor allem die drei folgenden Hauptziele verfolgt:

- langfristige Versorgungssicherheit gewährleisten;

- wirtschaftliche Effizienz steigern;

- Marktintegration von erneuerbaren Energien stärken.

In der Vorlage ist insbesondere die vollständige Marktöffnung des Strommarktes vorgesehen und die Optimierung der Netzregulierung, sprich die Erhöhung der Verursachergerechtigkeit, der Effizienz und der Transparenz.

Weiterhin ist der Verteilnetzbetreiber verpflichtet, sämtliche Flexibilitäten der Endverbraucherinnen und -verbraucher in Form eines Entgelts zu vergüten, das heißt der Verzicht auf eine große Leistungsspitze während einer bestimmten Zeitperiode, beispielsweise während der Mittagszeit oder das Steuern von Warmwassererzeugern (Boiler) oder anderer Verbraucher, muss durch den Verteilnetzbetreiber vergütet werden. Die Branche ist sich hierbei allerdings nicht sicher (VSE, Verband Schweizerische Elektrizitätsunternehmen 2019), wie das zukünftige Netznutzungsmodell aussehen wird, beziehungsweise wie sich die Netznutzungstarife verändern werden und wie der Stromverbrauch möglichst netzdienlich gesteuert werden kann. Auch im Hinblick auf die zunehmende Dezentralität 
und Elektromobilität spielen diese offenen Fragen eine wichtige Rolle. Bereits heute haben sich gemäß den Experten aus dem Verteilnetzbetrieb die Leistungsspitzen verschoben und das Netzverhalten der Endverbraucherinnen und -verbraucher verändert.

\subsection{Definitionen und Begriffserklärung}

Im folgenden Kapitel werden die wichtigsten Begriffe und Modelle im Zusammenhang mit dem Anwendungsfall erklärt. Die Blockchain-Technologie ist in aller Munde und wird größtenteils mit dem Bitcoin in Verbindung gebracht. Allerdings ist die Blockchain nur ein Teil innerhalb des Bitcoin-Ökosystems und stellt die notwendigen technischen Anforderungen zur Verfügung. Infolgedessen ist der Bitcoin lediglich eine Anwendung der vielfältigen Technologie. Die Blockchain basiert grundsätzlich auf dem Konzept der Distributed-Ledger-Technologien und birgt weitere interessante Ausprägungen.

\subsubsection{Distributed-Ledger-Technologien}

Die Distributed-Ledger-Technologie (DLT), stellt eine dezentrale Datenbank dar, welche es ermöglicht, ohne einen zentralen Server Daten zu verarbeiten und abzuspeichern. Die DLT besteht aus mehreren einzelnen Rechnern, den sogenannten Nodes, welche jeweils eine Kopie der gesamten Datensätze lokal abspeichern. Mit der Bildung eines Konsenses der Netzwerkteilnehmerinnen und -teilnehmer können weitere Datensätze hinzugefügt oder Transaktionen durchgeführt werden. Die daraus entstehenden Datensätze werden wiederum von den sogenannten Nodes lokal abgespeichert (Richard 2019, S. 29-31).

\subsubsection{Blockchain}

Die Blockchain ist die bekannteste Ausprägung der Distributed-Ledger-Technologie. Hierbei werden alle Datensätze resp. Transaktionen mittels der Kryptografie miteinander verkettet. Die Datensätze werden hierfür in verschiedenen Blocks zusammengeführt. Da nicht jede DLT in Blöcke zusammengeführt wird, ist demzufolge auch nicht jede DLT eine Blockchain. Umgekehrt gehört aber jede Blockchain zu den Distributed-Ledger-Technologien. In dieser Arbeit wird die Blockchain als gesamtes Ökosystem angeschaut. Dies beinhaltet zum einen die Blockgenerierung sowie die Art und Weise, wie der Konsens innerhalb des Netzwerks zustande kommt. Zum anderen umfasst die Blockchain auch die jeweiligen Akteure und deren Berechtigungen (Richard 2019, S. 29-31).

\subsubsection{Konsensfindung}

In einem dezentralen Netzwerk gibt es weder eine Geschäftsführung noch eine öffentliche Instanz, welche für alle Teilnehmer Entscheidungen trifft. Deswegen müssen Entschei- 
dungen, um beispielsweise eine Transaktion zu validieren und freizugeben, im Konsens des gesamten Netzwerks getroffen werden. Dies kann mit unterschiedlichen Methoden durchgeführt werden. In diesem Beitrag werden die zurzeit drei wichtigsten Verfahren beschrieben: Proof-of-Work, Proof-of-Stake und Proof-of-Authority (Peter 2017, S. 19).

\subsection{Proof-of-Work}

Die wohl bekannteste und auch von Bitcoin angewendete Methode ist die sogenannte Proof-of-Work-Konsensfindung. Diese Art von Konsensfindung geht von einem hohen Vertrauensmissbrauch aus und generiert eine große Last hinsichtlich Rechnerleistung. Aus diesem Grund werden durch Verwendung dieser Konsensfindung vergleichsweise große Mengen an Energie und Ressourcen verbraucht. Bei diesem Konzept versuchen sogenannte Miner des Netzwerks eine komplexe Rechnungsaufgabe in kurzer Zeit zu lösen, auch bekannt als Mining. Der erste Miner, welcher die Aufgabe korrekt gelöst hat, wird in Form von der jeweiligen Kryptowährung belohnt. Beim Bitcoin wird diese Belohnung alle 210.000 Blöcke halbiert. Mit der Proof-of-Work-Berechnung versuchen die Miner, eine bestimmte Transaktion zu validieren und die Korrektheit zu überprüfen. Dies wird mit den sogenannten Hash-Funktionen durchgeführt, welche schließlich einen Hash-Wert generieren. Ein Hash-Wert reduziert eine große Datenmenge auf eine kleinere Zeichenfolge. Ist die Berechnung erfolgt, werden die validierten Transaktionen zu einem Block zusammengefasst und der Blockchain angehängt. Peter (2017, S. 19) beschreibt das dem Proof-ofWork-Verfahren zugrunde liegende Prinzip und die Vertrauensmotivation aus Sicht des Miners folgendermaßen:

„Ich habe gearbeitet, um die Transaktion zu validieren. Dabei war ich schneller und daher besser als meine Konkurrenten. Die Lösung ist richtig und dafür erhalte ich Geld. Wieso sollte ich einen falschen oder manipulierten Block generieren? Dann würde ich ja nichts an meiner Arbeit verdienen."

Da sich sämtliche Miner im Netzwerk mit der Berechnung der Aufgabe resp. mit der Hash-Funktion beschäftigen, liegt das Vertrauen nicht bei einer einzelnen Gruppe oder einer Person, sondern im gesamten Netzwerk. Dies verringert die Chance eines Missbrauchs. Allerdings zeichnen sich der hohe Energieverbrauch und die relativ geringe Geschwindigkeit der Blockgenerierung als Nachteil aus.

\subsection{Proof-of-Stake}

Im Gegensatz zum Proof-of-Work-Verfahren benötigt das Proof-of-Stake-Verfahren weniger Energie und soll die Blöcke schneller generieren. Hierbei werden die Miner aufgrund des Vermögens gewichtet. Das Vermögen richtet sich nach dem Anteil der Token, welcher ein Miner besitzt. Im Zufallsverfahren wird schließlich ein Miner bestimmt, welcher die Aufgabe löst. Der Anteil der Tokens erhöht hierfür die Wahrscheinlichkeit, dass der Miner gewählt wird. Der Nachteil dabei ist, dass Teilnehmerinnen und Teilnehmer mit größeren Mengen an Tokens bevorzugt werden und die Chance, einen Block zu generieren, höher 
ist als bei Teilnehmerinnen und Teilnehmern mit einem kleineren Anteil. Peter (2017, S. 19) erläutert die Sicht des Miners folgendermaßen:

„Ich habe sehr viel in diese Blockchain investiert, ihr Fortbestand und ihre Funktionsfähigkeit sind mir wichtig, daher kannst du mir vertrauen. Ich baue dir einen neuen Block und bürge mit meinem in diese Blockchain investierten Vermögen für die Richtigkeit. Sollte der Block falsch oder manipuliert sein, verliere ich mein Vermögen“"

\subsection{Proof-of-Authority}

Die Proof-of-Authority-Konsensfindung knüpft an der Proof-of-Stake-Methode an und wurde im März 2017 entwickelt. Beim Proof-of-Authority gibt es keine herkömmlichen Miner, sondern die sogenannten Validatoren. Nebst der Anzahl an Coins und Tokens wird auch die Reputation der Validatoren bewertet. Beim Proof-of-Authority-Verfahren gibt es nur eine begrenzte Anzahl an Validatoren, welche einen Bewerbungsprozess durchlaufen müssen. Folgende Punkte werden dabei untersucht und spielen eine zentrale Rolle:

- Identität mit einer öffentlichen Datenbank überprüfen

- Gründliche Untersuchung der potenziellen Validatoren

- Überprüfung der finanziellen Lage und der Reputation der Validatoren

Für das Vertrauen der Netzwerkteilnehmerinnen und -teilnehmer erläutert Peter (2017, S. 19) die Sicht der Validatoren folgendermaßen:

„Bei der Konzeption dieser Blockchain wurde ich als Autorität festgelegt. Du kennst mich und vertraust mir. Wieso sollte ich also falsche Blöcke erzeugen?“

\subsubsection{Blockchain-Arten}

Die nachfolgend besprochenen Ausprägungen der Blockchain stellen vor allem den Grad der Dezentralität dar und haben ebenfalls einen Zusammenhang mit dem Konsensmechanismus. Das heißt, die Verteilung der Datenblöcke im Netzwerk und die Konsensfindung von einzelnen Entscheidungen. Dabei wird zwischen den öffentlichen, den privaten und den konsortialen Blockchains differenziert. Die öffentlichen Blockchains haben unbeschränkten Zugang und kommen mit dem Proof-of-Work-Verfahren zu einem Konsens. Die Datenblöcke werden auf der ganzen Welt durch unbegrenzt teilnehmende Nodes und Miners abgespeichert. Jeder kann sich einen entsprechenden Client kaufen und sämtlichen Datensatz lokal abspeichern. Infolgedessen spiegelt diese Art von Blockchain ein hohes Sicherheitsniveau in Bezug auf die Änderungen und Integrität der Daten sowie die Nachvollziehbarkeit wider.

Wie der Name sagt, bezieht sich eine private Blockchain auf einen geschlossenen Teilnehmerinnen- und Teilnehmerkreis, beispielsweise einer Unternehmung oder eines vordefinierten Kreises. Dadurch können auch Anwendungen mit sensiblen Daten schnell und flexibel eingesetzt werden. Zudem ist es möglich, verschiedene Perioden abzuschließen und zu archivieren. Dies führt dazu, dass sich die Datenmengen verringern und die Blockchain folglich durch mehr Kapazität und weniger benötigte Rechnerleistung schneller wird. 
Eine weitere Art ist die sogenannte konsortiale Blockchain als Mischform zwischen einer privaten und öffentlichen Blockchain. Sie kann beispielsweise durch den Zusammenschluss mehrerer Firmen entstehen. Die Ausprägung kann sich an eine bestimmte Anwendung oder Branche richten. Der Vorteil besteht darin, daß ein bestimmter Teilnehmerkreis, wie beispielsweise die Energiebranche, abgeschirmten Zugriff auf die Blockchain habt und die Art und Weise der Konsensfindung maßgebend bestimmt.

\subsubsection{Netznutzungsmodell}

Im Zusammenhang mit dem später beschriebenen Anwendungsfall, welcher zur Vereinfachung des zukünftigen Netznutzungsmodells beitragen soll, wird in diesem Kapitel näher auf das bestehende Modell eingegangen.

Das Netznutzungsmodell der Strombranche Schweiz NNMV-CH 2018 (VSE, Verband Schweizerischer Elektrizitätsunternehmen 2018) verfolgt vor allem zwei Ziele. Zum einen stellt dieses Modell sicher, dass die Nutzung der Netze diskriminierungsfrei ist. Jeder hat das Recht auf den Bezug von Elektrizität und muss für die von ihm verursachten Kosten einen verursachergerechten Beitrag leisten. Zum anderen muss das Modell dafür sorgen, dass die nötigen Mittel für den Betrieb, Instandhaltung, Ersatz und Ausbau für eine netzseitig hohe Versorgungsqualität sichergestellt sind. Dies wird mit der Erhebung der sogenannten Netznutzungsentgelte (siehe Abschn. 3.3.1) realisiert. Aus volkswirtschaftlichem Interesse sollen diese Entgelte möglichst tief gehalten werden, weswegen parallele Leitungsführungen und Anlagen zu vermeiden sind. Die Beurteilung der Netzkapazität basiert grundsätzlich auf der Aus- beziehungsweise Einspeisung von elektrischer Energie (VSE, Ver-band Schweizerischer Elektrizitätsunternehmen 2018, S. 12). Im Zusammenhang mit dem Netznutzungsmodell werden nach dem Branchendokument „Netznutzungsmodell für das schweizerische Verteilnetz" (VSE, Verband Schweizerischer Elektrizitätsunternehmen 2018, S. 13-14) und dem Branchendokument „Anbindung von Regelpools an den Schweizer SDL-Markt“ (VSE, Verband Schweizerischer Elektrizitätsunternehmen 2013, S. 8-10) sowie dem Netznutzungsmodell Swissgrid (swissgrid ag 2012, S. 10) verschiedene Marktakteure unterschieden, welche nachfolgend kurz erläutert werden.

\section{Verteilnetzbetreiber}

Der Verteilnetzbetreiber (VNB) ist verantwortlich für den sicheren, zuverlässigen und leistungsfähigen Betrieb des ihm zugeordneten Verteilnetzes, welches durch den jeweiligen Kanton bestimmt wird. Er übernimmt dabei auch die Verantwortung über die nichtdiskriminierende Bereitstellung des Verteilnetzes und über das Mess- und Informationswesen.

\section{Übertragungsnetzbetreiber}

Der Übertragungsnetzbetreiber (ÜNB) stellt die nationale Netzgesellschaft (Swissgrid) dar, welche gleichermaßen über das Übertragungsnetz verantwortlich ist. 


\section{Energielieferant}

Der Energielieferant speist elektrische Energie ins Netz ein und versorgt damit die Endverbraucherinnen und -verbraucher. Vielfach wird hier von einem Energieversorgungsunternehmen (EVU) gesprochen. Beispielsweise ist Energie Wasser Bern einerseits ein EVU, welches elektrische Energie bereitstellt. Andererseits ist das Unternehmen auch ein VNB, welcher für die Netzbewirtschaftung der Stadt Bern zuständig ist.

\section{Endverbraucher}

Endverbraucherinnen und -verbraucher nutzen im Sinne des VNB die Netzinfrastruktur und müssen dem VNB ein Netznutzungsentgelt für die verursachten Kosten entrichten.

\section{Prosumer}

Ein Prosumer ist eine Endverbraucherin bzw. ein Endverbraucher, welcher zum einen elektrische Energie über das Netz bezieht und zum anderen elektrische Energie selbst produziert und an Ort und Stelle verbraucht oder ins Netz einspeist.

\section{Erzeuger}

Eine Erzeugerin oder ein Erzeuger betreibt eine oder mehrere Erzeugungseinheiten (EZE) für elektrische Energie, welche entweder am Ort der Produktion selbst verbraucht oder ins Netz eingespeist wird.

\section{Speicherbetreiber}

Ein Speicherbetreiber bezieht elektrische Energie aus dem Netz, speichert diese und gibt sie zu einem späteren Zeitpunkt wieder ans Netz ab.

\section{Regelpoolbetreiber}

Der Regelpoolbetreiber ist für einen Pool von beliebig vielen EZE, die Regelleistung vorbehalten und bei Bedarf Regelenergie liefern können, verantwortlich. Die Übermittlung der notwendigen Daten und Informationen sämtlicher beteiligten Energieerzeugungsanlagen (EZA) an den ÜNB ist ebenfalls in seiner Verantwortung.

\section{Bilanzgruppenverantwortlicher}

Der Bilanzgruppenverantwortliche (BGV) bewirtschaftet ein ihm zugeordnetes Portfolio mit Messpunkten von Endverbraucherinnen und -verbrauchern, Prosumern sowie EZA und weiteren Kraftwerken. Er ist für die fristgerechte Übermittlung von Fahrplänen sowie für das Sicherstellen der Energiebilanz gegenüber dem ÜNB verantwortlich. Dabei müssen Verbrauch und Produktion immer im Gleichgewicht sein.

\subsubsection{Netznutzungsentgelt}

Das Netznutzungsmodell beinhaltet unter anderem auch das Netznutzungsentgelt, welches durch den geografisch zuständigen Verteilnetzbetreiber erhoben wird. In diesem Kapitel sollen nun die wichtigsten Anforderungen beziehungsweise die übergeordneten Ziele 
des Netznutzungsentgelts betrachtet werden. Das Netznutzungsentgelt wird insgesamt in vier Komponenten unterteilt, nämlich in den Netznutzungstarif, die Systemdienstleistungen, die Abgaben an das Gemeinwesen und in die Bundesabgaben (siehe Abschn. 3.3.2). Die Netznutzungsentgelte müssen verursachergerecht und diskriminierungsfrei sein. Die anfallende Netzbelastung und die daraus entstehenden Kosten sollen durch die jeweilige Verursacherin bzw. den Verursacher getragen werden. Weiter sollen sie zur Energieeffizienz und zur Ressourcenschonung beitragen sowie dezentrale Energieerzeugungsanlagen integrieren. Die Netznutzungsentgelte spiegeln ebenfalls einen Steuerungsmechanismus im Sinne einer Anreizschaffung zu netzdienlichem Verhalten wider. Dieses soll zur Effizienz beitragen, wodurch unnötige Netzausbauten verhindert werden können. Besonders zu beachten ist, dass die Entgelte einfache und verständliche Strukturen aufweisen müssen (Eidgenössische Elektrizitätskommission ElCom 2019).

\subsubsection{Stromtarifierung}

Der größte Teil der Schweizer VNB bestimmt seine Stromtarife jährlich und muss diese immer per 31. August für das folgende Jahr publizieren (Eidgenössische Elektrizitätskommission ElCom 2019). Zudem sind die VNB verpflichtet, deren Bestandteile jährlich anhand von standardisierten Berichten dem Regulator ElCom zu kommunizieren. Die zu übermittelnden Hauptbestandteile sind die Herleitung der anrechenbaren Kosten für die Bestimmung der Netznutzungsentgelte, die Kalkulation der Gestehungskosten für die Energie und die Kalkulation der Tarife. Die Tarifierung erfolgt jeweils auf der Datenbasis des vergangenen Jahres.

Der Stromtarif besteht aus den fünf Komponenten Netznutzungstarif, Energieliefertarif, Systemdienstleistungen, Abgaben und Leistungen an das Gemeinwesen sowie die Bundesabgaben. Der Netznutzungstarif wird für den Energietransport vom Ort der Produktion bis zum Verbraucher erhoben. Diese Komponente beinhaltet vor allem die kalkulatorischen Kapitalkosten der Netzinfrastruktur des jeweiligen VNB und sämtliche Kosten, welche durch den Transport anfallen. Die Netzinfrastrukturrendite ist reguliert und wird mit dem vorgegebenen WACC berechnet. Der Energieliefertarif ist der eigentliche Anteil, der bei einer vollkommenen Marktöffnung von der Endverbraucherin bzw. dem Endverbraucher frei ausgewählt werden kann. Er spiegelt die tatsächliche Energielieferung wider. Zurzeit wird dieser allerdings aufgrund der Gestehungskosten und den Verwaltungs- und Vertriebsgemeinkosten (VVGK) sowie einem regulierten Gewinnanteil berechnet. Sobald der Markt auch für Kleinkundeninnen und -kunden ( $<100 \mathrm{MWh})$ geöffnet ist, kann dieser Preis frei durch das EVU bestimmt werden resp. er wird sich dem Markt anpassen müssen. Für den ständigen Ausgleich von Verbrauch und Produktion, resp. um die Netzstabilität zu garantieren, werden vom übergeordneten Netzbetreiber (Swissgrid) die sogenannten Systemdienstleistungen erhoben. Unter den Abgaben und Leistungen an das Gemeinwesen sind die kantonalen und kommunalen Beiträge zu verstehen, welche für allgemeine Leistungen wie beispielsweise Konzessionsgebühren für die Sondernutzung von öffentlichem Grund erhoben werden. Gemäß Art. 35ff. der Energieverordnung wird ein allgemeiner Netzzuschlag für den Bund erhoben, welcher im Stromtarif als Bundesabgabe gekennzeichnet ist. 
Im Jahr 2019 betrug der Stromtarif des Netznutzungsgebietes von Energie Wasser Bern mit dem Energielieferprodukt „ewb.NATUR.Strom“ und der Netznutzungskategorie „Home“, welche dem Einfachtarif entspricht, gerundet insgesamt 21,50 Rp/kWh. (Eidgenössische Elektrizitätskommission ElCom 2020) Davon gehen 7,70 Rp/kWh zulasten des Netznutzungstarifs und lediglich 8,60 Rp/kWh zulasten des Energieliefertarifs. Die restlichen 5,20 Rp/kWh müssen dem übergeordneten Verteilnetzbetreiber, dem Gemeinwesen sowie dem Bund vergütet werden.

Die Öffnung des Strommarktes würde bedeuten, dass jede Endverbraucherin bzw. jeder Endverbraucher und Prosumer den Lieferanten für den Energieteil frei wählen könnte, sprich im vorherigen Beispiel wären dies die 8,60 Rp/kWh. Die vier anderen Komponenten bleiben im regulierten Bereich und werden durch den geografisch zuständigen Verteilnetzbetreiber erhoben.

Der Beitrag sowie der folgende Anwendungsfall fokussiert sich vor allem auf den regulierten Teil des gesamten Stromtarifs.

\subsubsection{Ein Blick in die Zukunft}

Grundsätzlich ist die Gestaltung von flexiblen Netznutzungstarifen gemäß der ElCom Mitteilung vom 27.02.2019 (Eidgenössische Elektrizitätskommission ElCom 2019) zulässig. Das heißt, neben einem Einfachtarif darf der Verteilnetzbetreiber dem Endkunden verschiedene Wahltarife anbieten, welche beispielsweise das netzdienliche Verhalten fördern sollen. Die Zuordnung eines Wahltarifs erfolgt dabei immer im gegenseitigen Einverständnis. Sämtliche Tarife müssen allerdings stets den Kriterien aus dem Abschn. 3.3.1 entsprechen. Deswegen müssen Regeln, wie ein Tarif zustande kommt, vorher definiert und klar kommuniziert werden. Dabei sind Flexibilitäten, welche von der Endverbraucherin bzw. dem Endverbraucher oder Prosumer zur Verfügung gestellt werden, in Form eines Entgelts zu entrichten (Eidgenössische Elektrizitätskommission ElCom 2019). Zusammengefasst eröffnet sich für den Verteilnetzbetreiber ein großer Handlungsraum, wie er seine Netznutzungstarife gestaltet und wie er seine Endverbraucherinnen bzw. -verbraucher und Prosumer dazu motivieren kann, das Netz möglichst optimal zu belasten. Diese Faktoren und die beschriebenen übergeordneten Ziele aus dem Abschn. 3.3.1 stellen die gesamte Strombranche vor diverse Herausforderungen. Einerseits ist der Verteilnetzbetreiber gewillt, die Endverbraucherinnen bzw. -verbraucher und Prosumer mit unterschiedlichen Wahltarifen zu steuern, damit Engpässe vermieden werden können. Andererseits ist er verpflichtet, die Strukturen der Tarifierung einfach und verständlich zu halten. Vom Regulator wird ebenfalls ein effizienter Netzbetrieb gefordert, was effiziente Prozesse impliziert. Des Weiteren sind in der Schweiz über 600 VNB zu verzeichnen, wodurch eine vielfältige Palette aus kreativen Netznutzungstarifen entstehen könnte. Diesbezüglich besteht die Gefahr eines unübersichtlichen Tarifdschungels. Auch im Hinblick auf die Strommarktöffnung und die vermehrte Dezentralisierung der Produktion wird sich die Abwicklung der Verrechnung und des Datenmanagements vermehrt erschweren. 
Die DLT resp. Blockchain könnte hier eine mögliche Lösung darstellen, wie solche Wahltarife, das netzdienliche Verhalten und das Datenmanagement abgewickelt bzw. abgebildet werden könnten. Automatische Abläufe könnten zudem zur Verbesserung der Prozesseffizienz beitragen.

\subsection{Anwendungsfall für den Verteilnetzbetreiber}

In den folgenden Abschnitten wird nun eine mögliche Anwendung der beschriebenen Technologie im heutigen Umfeld des Netzbetriebs beschrieben.

\subsubsection{Derzeitige Optimierungsmöglichkeiten}

In diesem Abschnitt sollen der Leserin und dem Leser die Optimierungsmöglichkeiten des Netznutzungsmodells, unter anderem der Meter-to-Cash(M2C)-Prozess, die flexible Tarifierung sowie der Informationsaustausch der Marktakteure nähergebracht werden. In Abb. 3.1 ist der M2C-Prozess und die damit verknüpfte Verrechnung und Tarifierung zu erkennen.

Bis 2027 sind die Verteilnetzbetreiber verpflichtet, mindestens $80 \%$ ihrer Messpunkte mit einem Smartmeter auszurüsten. Dieser sendet alle 15 min einen Lastgang an den Verteilnetzbetreiber des jeweiligen Messpunktes. Zurzeit ist allerdings unklar, wo diese Daten gespeichert werden sollen und welche Instanz die Datenhoheit darüber ausüben soll. Des Weiteren generiert die periodische Segmentüberprüfung Aufwand, welcher nicht zu vermeiden ist. Das heißt, um festzustellen, ob sich die Kunden im richtigen Netznutzungstarif befinden oder nicht, müssen dies die Verteilnetzbetreiber basierend auf den aktuellen Messdaten kontrollieren und bei Bedarf eine Umtarifierung vornehmen. Dies generiert zum einen Aufwand bei der laufenden Beobachtung, zum anderen bei der eigentlichen,

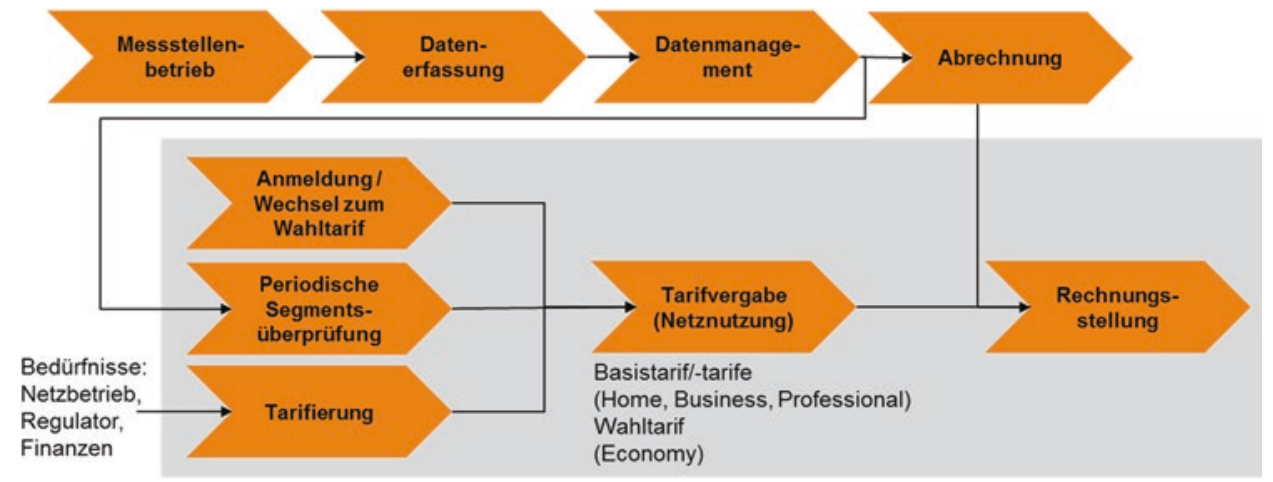

Abb. 3.1 M2C-Prozess 
zurzeit noch manuellen Umtarifierung. Eine weitere Schwierigkeit stellen die im Abschn. 2.2 beschriebenen Wahltarife bzw. Vergütung von Flexibilitäten dar. Ein Wahltarif darf nur mit schriftlichem Einverständnis der beiden Parteien vergeben werden. Auch dies hat einen erhöhten Aufwand zur Folge. Der Regulator will außerdem die volle Transparenz über alle Tarife und deren Entstehung erhalten.

Innerhalb des Netznutzungsmodells agieren verschiedene Marktakteure, welche unterschiedliche Informationen austauschen. Dieser Informationsaustausch, welcher teilweise mit dem sogenannten Wechselprozess verknüpft ist, ist kompliziert und generiert ebenfalls einen hohen Aufwand. Nachfolgend sind die Handlungsfelder und die Bedürfnisse der jeweiligen Marktakteure sowie das Potenzial einer Vereinfachung dargelegt. Der Regulator und der Speicherbetreiber sind aufgrund deren Interessen ergänzt bzw. weggelassen worden. Überdies werden die Interessen zwischen der Erzeugerin bzw. dem Erzeuger und dem Prosumer zusammengefasst.

\section{Übertragunsnetzbetreiber (Swissgrid)}

Die Kapazitätsplanung des Übertragungsnetzes erfordert eine möglichst genaue Prognose sämtlicher Verteilnetzbetreiber. Eine einheitliche Plattform könnte hier zur Prozessoptimierung beitragen.

\section{Bilanzgruppenverantwortlicher}

Die Messdaten und Informationen der einzelnen Messpunkte müssen durch den Bilanzgruppenverantwortlichen (BGV) eingeholt und dem Übertragungsnetzbetreiber weitergeleitet werden. Eine einheitliche Plattform könnte auch hier zur Prozessoptimierung beitragen.

\section{Regelpoolbetreiber}

Die Daten und Informationen der beteiligten Energieerzeugungsanlagen müssen dem Übertragungsnetzbetreiber übermittelt werden. Auch hier würde eine einheitliche Plattform eine Vereinfachung ermöglichen.

\section{Verteilnetzbetreiber}

Der Verteilnetzbetreiber (VNB) ist für die Erhebung und für den Austausch der Meß- sowie Kundendaten zuständig. Dies impliziert, auch durch die unterschiedlichen Systemlandschaften, zurzeit einen großen Aufwand. Zudem muss der VNB für einen Wahltarif das Einverständnis des jeweiligen Kunden einholen, was ebenfalls zu erhöhtem Aufwand führt. Im Hinblick auf die Strommarktliberalisierung sollen die Wechselprozesse möglichst effizient gestaltet und durchgeführt werden können. Dabei spielen allerdings auch das Datenmanagement und der Umgang mit dem Datenschutz eine zentrale Rolle. Zusätzlich benötigt der VNB ein Instrument, wie er seine Kunden zu einem netzdienlichen Verhalten fördern kann, damit unnötige Netzausbauten verhindert werden können. 


\section{Energielieferant}

Die Wechselprozesse bzw. die Energielieferantenwechsel können ohne die Zustimmung vom VNB nicht durchgeführt werden. Auch der Energielieferant ist an effizienten Prozessen interessiert. Zudem wäre eine Vereinheitlichung der Plattformen von Vorteil.

\section{Endverbraucher}

In der Schweiz gibt es über 600 VNB, wodurch die Gefahr besteht, dass es unterschiedliche Tarifstrukturen gibt resp. geben könnte. Dadurch könnte sich ein unübersichtlicher Tarifdschungel entwickeln. Die Endverbraucherin bzw. der Endverbraucher möchte hauptsächlich günstige Tarife mit einfachen und nachvollziehbaren Strukturen.

\section{Prosumer}

Wie bei der Endverbraucherin bzw. dem Endverbrauchern besteht auch hier die Gefahr von einem Tarifdschungel. Der Prosumer möchte primär günstige Ein- und Ausspeisetarife mit einfachen Strukturen. Des Weiteren strebt er an, die überschüssige Energie mit möglichst wenig Aufwand ins Netz einzuspeisen und dafür eine Vergütung zu erhalten.

\section{Regulator (EICom)}

Die Tarife sowie die Grundlagen zu deren Berechnung werden in einem aufwendigen Prozess vom Regulator überprüft und bei Bedarf vom VNB überarbeitet. Der Regulator möchte die volle Transparenz in Bezug auf die Tarife und deren Entstehung. Zusätzlich fordert er einen effizienten Netzbetrieb.

\subsubsection{Lösungsvorschlag zur Verbesserung}

Der Anwendungsfall „Blockchain for grid applications“ zeigt erste Ansätze auf, welche zur Optimierung der Prozesse innerhalb des Netznutzungsmodells beitragen. Er basiert auf der Technologie der Blockchain. „Blockchain for grid applications“ bildet nicht nur die Verrechnung der Netznutzungstarife auf dem Smart Contract ab, sondern zeigt ebenfalls auf, wie die Blockchain das Datenmanagement bezüglich der Messdaten und die Abwicklung der Wechselprozesse unterstützen könnte. Der Anwendungsfall ist in Abb. 3.2 dargestellt. Nachfolgend werden nun die Teilnehmerinnen und Teilnehmer sowie die Tätigkeiten beschrieben.

Die Registrationen eines neuen Smart Meters, beispielsweise im Zusammenhang mit einem Neubau, erfolgt durch den zuständigen VNB. Dieser weist die entsprechende Smart-Meter-ID dem neuen Messpunkt zu und ermittelt die korrespondierenden Stammdaten. Die Daten werden anschließend in die Blockchain geschrieben. Für die Bestimmung der Tarifvarianten (Einfachtarife und/oder Wahltarife) und der Tarifhöhe (Tarifierung) können der Blockchain vergangenheitsbezogene Messdaten entnommen werden. Netzkosten, regulatorische sowie gesetzliche, und weitere Einflussfaktoren sind hier nicht aufgeführt, weshalb die Tarifierung als Subprozess gekennzeichnet ist. Nachdem der VNB die 

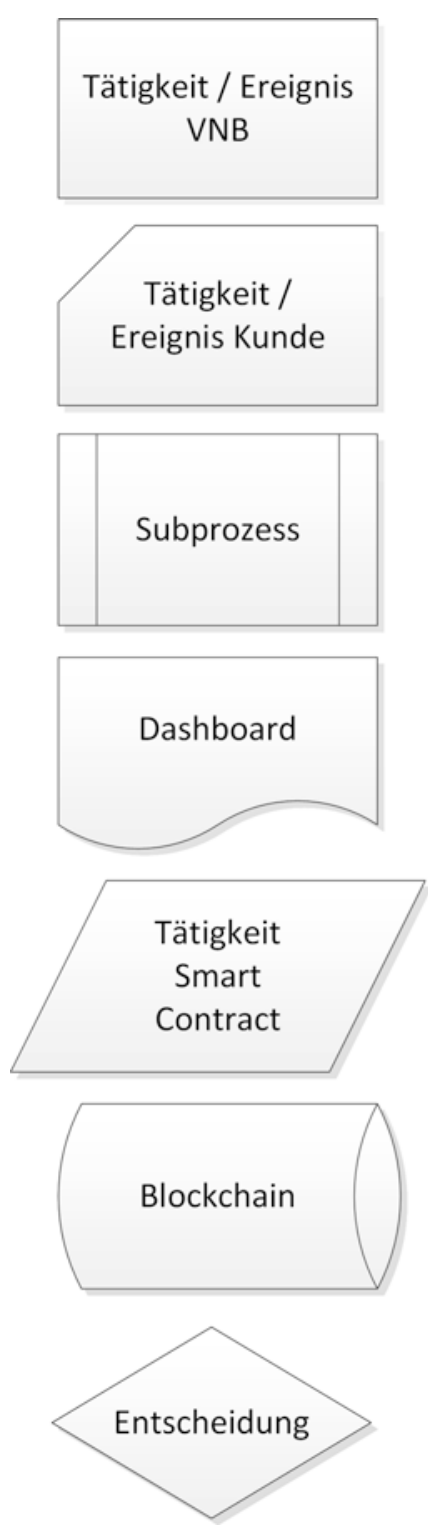

Dieses Symbol veranschaulicht sämtliche Tätigkeiten, welche beim Verteilernetzbetreiber (VNB) anfallen oder demonstriert ein Ereignis, beziehungsweise den Zahlungseingang beim VNB.

Anstatt des VNB bildet dieses Symbol die Tätigkeiten und Ereignisse des/der Netzkunden/-in ab. Darunter fallen vor allem die Endverbraucher/-innen und Prosumer.

Ein Subprozess beinhaltet mehrere Aktivitäten und weitere Prozesse. Diese Teilaktivitäten werden allerdings icht im Detail beschrieben.

Das Dashboard soll eine interaktive Plattform zwischen Kundenen und dem Verteilnetzbetreiber darstellen. Dabei können Informationen wie Messdaten, Fahrpläne, Tarifvarianten od. Rechnungen abgerufen werden. Zudem könnte das Dashboard für weitere Marktakteure erweitert werden. Beispielsweise für sämtliche Wechselprozesse.

Hiermit werden die Aktivitäten des Smart Contracts erläutert.

Dieses Symbol veranschaulicht die Blockchain-Plattform. Entweder können Daten abgerufen oder in die Blockchain geschrieben werden.

Mit diesem Symbol wird eine Entscheidung dargestellt.

Abb. 3.2 Legende der verwendeten Prozesssymbole

Tarifausprägungen und deren Höhe bestimmt hat, werden diese ebenfalls in die Blockchain geschrieben. Dies kann sowohl die gesetzlich vorgegebenen Einfachtarife als auch mehrere Wahltarife beinhalten. Die Endverbraucherin bzw. der Endverbraucher oder Prosumer hat nun die Möglichkeit, sich über die aktuellen Tarife im Dashboard zu informieren und ihren/seinen Verbrauch bei Bedarf entsprechend anzupassen und die zur Verfügung stehenden Tarifmöglichkeiten auszuwählen. Größere Endverbraucher müssen dem 
VNB einen Fahrplan melden, also zu welcher Zeit sie größere Mengen an Strom benötigen. Bei kleineren Endverbrauchern spiegelt der ausgewählte Tarif den geplanten Fahrplan wider. Beispielsweise möchte ein Endverbraucher seine Heizung möglichst während der günstigsten Tarifzeit einschalten. Dies gibt dem VNB und dem Energielieferanten die Möglichkeit, die Netzkapazitäten und Energielieferungen entsprechend zu planen und zu steuern. Des Weiteren soll der VNB die Möglichkeit haben, die Tarifvarianten auf den Energieverbrauch und die Anschlussleistung der Endverbraucherin bzw. des Endverbrauchers oder Prosumers zu beschränken. In Anbetracht der gesetzlichen Vorgaben steht dem VNB hiermit die Möglichkeit offen, unterschiedliche Kundengruppen zu bilden. Im Anschluss an den erstellten Fahrplan resp. nach der Tarifauswahl wird durch den Smart Contract automatisch eine Offerte erstellt, welche wiederum im Dashboard ersichtlich ist. Die Offerte basiert zudem auf historischen Messdaten sowie den entsprechenden Stammdaten und zeigt dem Endverbraucher oder Prosumer auf, mit wie vielen Netznutzungskosten er rechnen muss. Entweder ist der Endverbraucher oder Prosumer zufrieden und akzeptiert die Offerte, oder er überarbeitet seinen Fahrplan beziehungsweise seine Auswahl noch einmal. Die viertelstündigen Messdaten (Lastgangdaten) des Smart Meters werden laufend in die Blockchain geschrieben. Aufgrund dessen erfolgt schlussendlich der Soll/ Ist-Abgleich über den Smart Contract. Dieser vergleicht den anfangs eingegebenen Tarif resp. Fahrplan mit dem tatsächlichen Verbrauch und schreibt die Abweichung in die Blockchain. Im Anschluss daran löst der Smart Contract aufgrund der Messdaten und des Soll/Ist-Abgleichs eine Zahlung aus. Die Werte werden mittels „Energy“ Token (siehe Abb. 3.3 und 3.4) vom der Endverbraucher oder Prosumer zum VNB oder bei der Einspeisung von Energie ins Netz vom VNB zum Prosumer verschoben. Ein Token kann beispielsweise einem Wert von $10 \mathrm{Rp}$. entsprechen. Der Zeitpunkt für die definitive Verrechnung in CHF kann der VNB nun beliebig bestimmen. Nebst der Verrechnung der Netznutzungsentgelte sollen die Wechselprozesse über die Blockchain abgewickelt resp. durch einen Smart Contract unterstützt werden. Zum Beispiel müssen beim Wechsel eines Energielieferanten unterschiedliche Zugriffberechtigungen erteilt werden, damit die jeweiligen Marktakteure die notwendigen Informationen abrufen und Änderungen durchführen können. Die Blockchain-Plattform ermöglicht zudem eine einheitliche Datenbasis, welche mit unterschiedlichen Zugriffsberechtigungen den Marktakteuren erlaubt, die notwendigen Informationen abzurufen. Dabei spielt die Vergabe des öffentlichen Schlüssels eine zentrale Rolle. Die Vergabe von unterschiedlichen öffentlichen Schlüsseln ermöglicht, dass nicht alle Daten ohne Weiteres durch jede Teilnehmerin und jeden Teilnehmer abgerufen werden können.

In Abb. 3.5 ist ein mögliches Architekturmodell für den Anwendungsfall „Blockchain for grid applications“ dargestellt. Dieses illustriert den Aufbau und die Kommunikation sowie Interaktion zwischen den verschiedenen Komponenten und Teilnehmerinnen und Teilnehmern. Die Module und der Aufbau richten sich nach Stefan Wunderlich (2018, S. 1267) und sind nicht abschließend. Für die Umsetzung müssten hier weitere Abklärungen getroffen werden. Insbesondere im Hinblick auf die verwendete Blockchainl-Lösung und der Schnittstelle zum Smart Meter. 


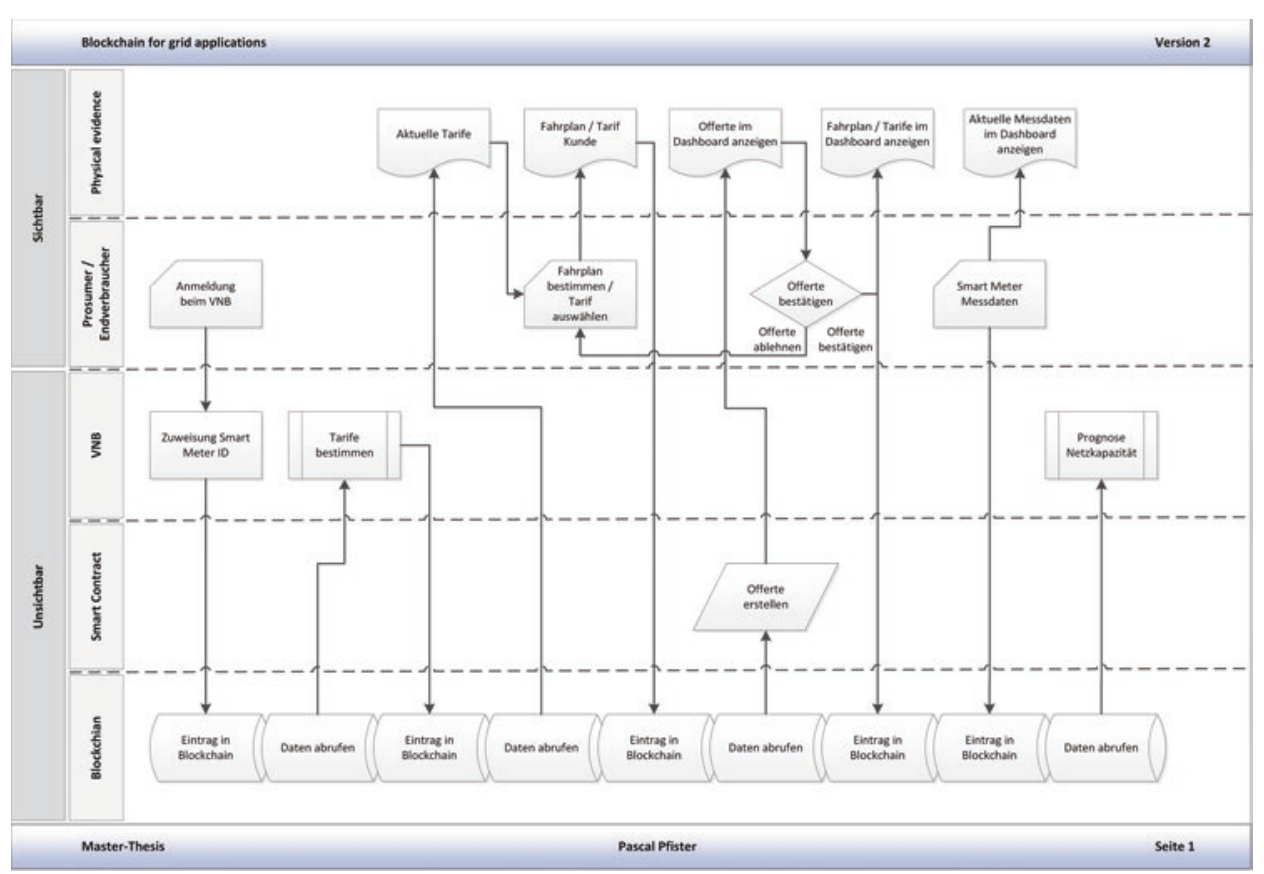

Abb. 3.3 Blockchain für Gridanwendungen (Teil 1)

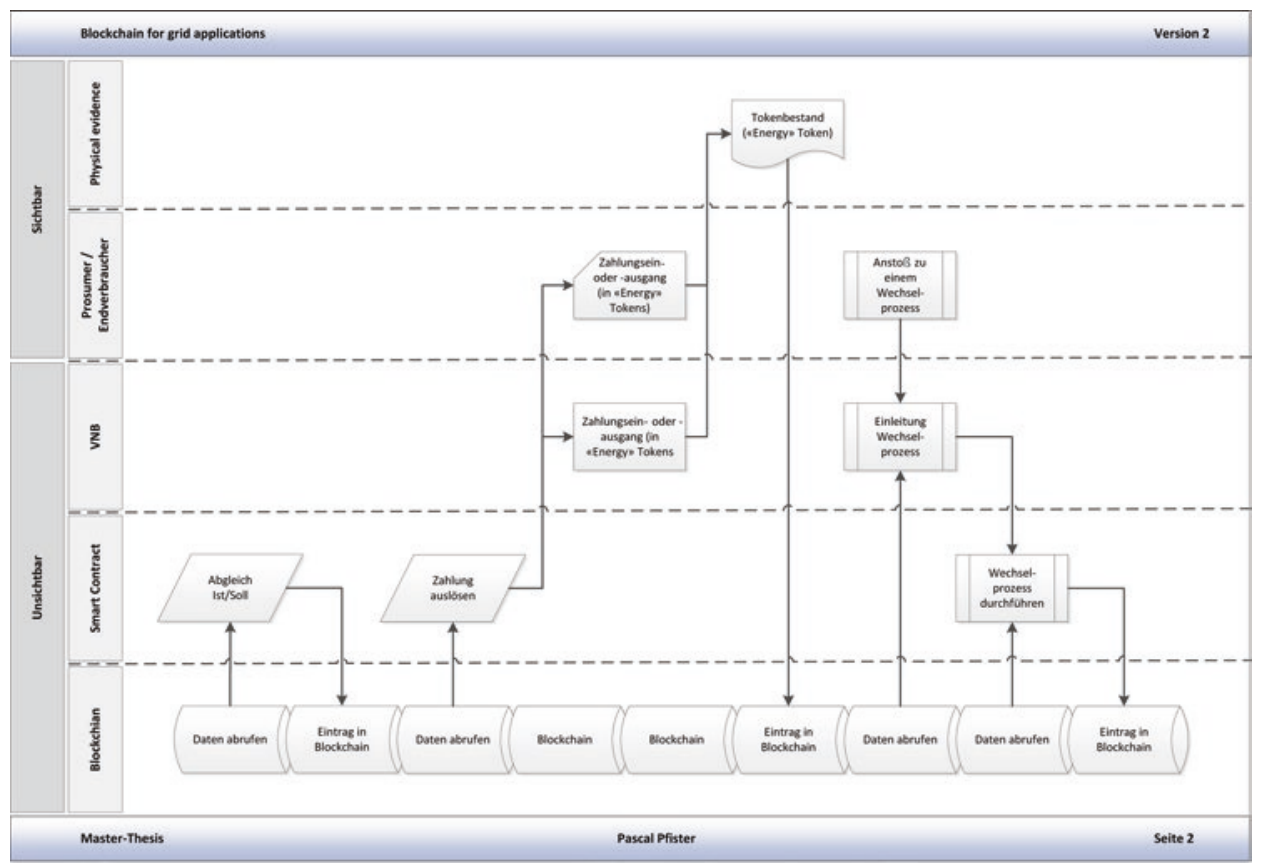

Abb. 3.4 Blockchain für Gridanwendungen (Teil 2) 


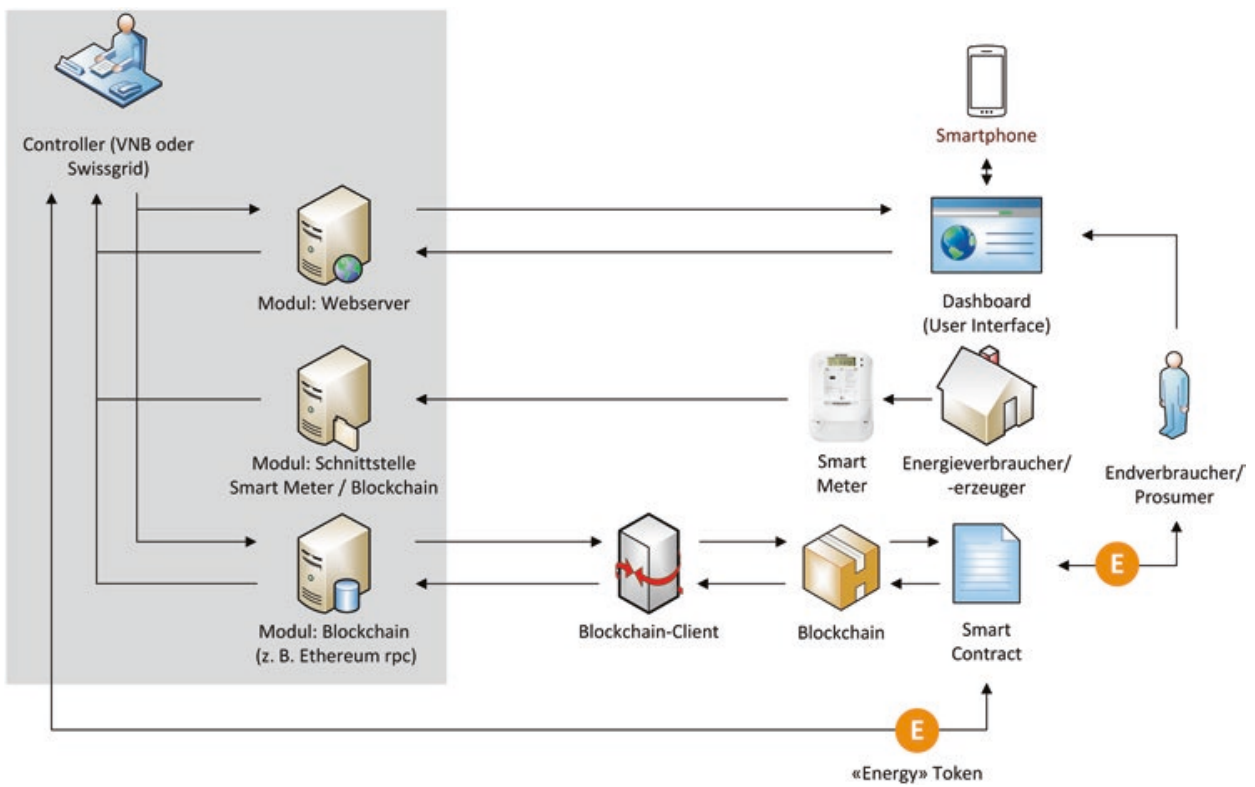

Abb. 3.5 Architekturmodell nach Wunderlich (2018)

Die Messdaten der Smart Meter werden automatisch in die Blockchain geschrieben und können jederzeit auf dem Dashboard angezeigt werden. Der Endverbraucher oder Prosumer kommuniziert über das Dashboard mit dem VNB. Hierfür wählt er den gewünschten Tarif und gibt seine Fahrpläne ein bzw. wählt die gewünschten Tarife aus. Zusätzlich kann der aktuelle Token-Kontostand abgerufen werden. Der Controller übernimmt eine Steuerungs- und Koordinationsfunktion. Diese sollte entweder beim VNB und/oder beim ÜNB (Swissgrid) liegen. Nebst der Interaktion mit der Kundin bzw. dem Kunden soll der Anwendungsfall eine einheitliche Plattform bilden, auf welcher die Messdaten zwischen den Marktakteuren resp. den Stakeholdern ausgetauscht werden können; beispielsweise zur Unterstützung der Wechselprozesse, bei welchen Informationen zwischen dem VNB, dem SDV, dem BGV, dem Energielieferanten und dem ÜNB ausgetauscht werden müssen.

\subsection{Validierung}

\subsubsection{Erkenntnisse aus der Empirie}

Für die Erarbeitung des Anwendungsfalls wurden im Rahmen eines Workshops Experten aus der Energiebranche hinzugezogen. Diese hatten unter anderem auch Erfahrungen im Zusammenhang mit der Anwendung der Blockchain im Energiesektor. Die anschließende Validierung ist mit einem Experten der Informationssicherheit und der Kryptografie, mit einem Experten der DLT- und Blockchain-Technologie sowie mit einem Experten der Tari- 
fierung und des Regulationsmanagements durchgeführt worden. Hierfür wurden drei Experteninterviews durchgeführt, welche in erster Linie die folgenden Themen beinhalteten:

- Vorteile der Blockchain und der Smart Contracts

- Merkmale der Blockchain und der Smart Contracts

- Herausforderungen und Risiken des Anwendungsfalls

- Kriterien der Blockchain in Bezug auf den Anwendungsfall

- Regulatorische Rahmenbedingungen

Die Erkenntnisse werden in den nachfolgenden Betrachtungen widergespiegelt.

\subsubsection{Technologische Betrachtung}

Im Workshop konnte bestätigt werden, dass für die Smart-Meter-Daten eine Datenbank benötigt wird, welche es ermöglicht, unterschiedliche Zugriffsrechte für die Stakeholder zu erteilen. Des Weiteren stellt der Verteilnetzbetreiber die zentrale Instanz bzw. verfügt über die Datenhoheit und genießt deswegen das Vertrauen der Endverbraucherinnen und -verbraucher. Dies konnte durch die Experteninterviews ebenfalls bestätigt werden. Die Plattform benötigt anpassbare Funktionalitäten, weswegen sich eine öffentliche Blockchain nicht eignet. Die Wechselprozesse erfordern eine Interaktion zwischen verschiedenen Marktakteuren und Unternehmen. Aus diesen Gründen sollte die Lösung auf einer konsortialen Blockchain basieren und kann beim konkreten Anwendungsfall durchaus Sinn ergeben. Die Konsensfindung müsste diesbezüglich entweder auf dem Proof-of-Authority- oder auf dem Proof-of-Stake-Verfahren basieren.

In Anlehnung an Höfelmann (2019) wurden vor dem Workshop durch den Autor Kriterien in Bezug auf die Blockchain bestimmt. Während des Workshops sind diese im Hinblick auf den Anwendungsfall „Blockchain for grid applications“ durch die Teilnehmer gewichtet und ergänzt worden. Die Kriterien sind für den Anwendungsfall entweder „,sehr wichtig“, „,neutral“ oder ,nicht wichtig“. In Tab. 3.1 sind sämtliche Kriterien und deren Gewichtung zu finden.

Bis auf die Installation, die Datenspeicherung und die Systemkosten muss die Blockchain-Lösung alle Kriterien erfüllen. Die langfristige Datenspeicherung über mehrere Jahre steht bei den Smart-Meter-Daten nicht im Vordergrund. Diese müssen grundlegend für die Verrechnung der Stromtarife und für den jährlichen Tarifierungsprozess zur Verfügung stehen. Weitere Verwendungszwecke sind nicht von besonderer Bedeutung. Die Systemkosten, also die Betriebskosten der Blockchain und deren IT-Infrastruktur, können über die kalkulatorischen Netzkosten auf sämtliche Endverbraucher und Prosumer abgewälzt werden, solange diese geringer als die bisherigen Verwaltungskosten ausfallen. Soll heißen, dass ein wirtschaftlicher Nutzen ersichtlich sein muss. Die Installation soll kein Hindernis darstellen, ob die Lösung umgesetzt wird oder nicht, deswegen wurde sie als 
Tab. 3.1 Kriterien für die Blockchain des Anwendungsfalls „Blockchain for grid applications“

\begin{tabular}{|c|c|c|c|}
\hline & 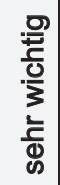 & $\begin{array}{l}\overline{\mathbb{d}} \\
\stackrel{5}{\bar{\Xi}} \\
\stackrel{\Phi}{c}\end{array}$ & $\begin{array}{l}\frac{0}{2} \\
\frac{5}{0} \\
\frac{0}{3} \\
\frac{5}{0} \\
\frac{0}{2}\end{array}$ \\
\hline \multicolumn{4}{|l|}{ Installation, Benutzerfreundlichkeit } \\
\hline \multirow{2}{*}{$\begin{array}{l}\text { - Dokumentation } \\
\text { - Installation }\end{array}$} & $x$ & & \\
\hline & & & $x$ \\
\hline \multicolumn{4}{|l|}{ Effizienz, Performance } \\
\hline \multirow{4}{*}{$\begin{array}{l}\text { - Datenvolumen pro Sekunde } \\
\text { - Datenspeicherung (langfristig) } \\
\text { - Transaktionsbestätigungszeit (z. B. Offerte) } \\
\text { - Hardware (Systemsicherheit) }\end{array}$} & $x$ & & \\
\hline & & & $x$ \\
\hline & $x$ & & \\
\hline & $X$ & & \\
\hline \multicolumn{4}{|l|}{ Kosteneffizienz } \\
\hline \multirow{2}{*}{$\begin{array}{l}\text { - Transaktionsgebühren } \\
\text { - Systemkosten }\end{array}$} & $x$ & & \\
\hline & & $x$ & \\
\hline \multicolumn{4}{|l|}{ Fähigkeit der Blockchain, Aktualität } \\
\hline \multirow{4}{*}{$\begin{array}{l}\text { - Reifegrad der Blockchain } \\
\text { - Upgrade-Fähigkeit } \\
\text { - Community } \\
\text { - Updates }\end{array}$} & $x$ & & \\
\hline & $x$ & & \\
\hline & $\mathrm{x}$ & & \\
\hline & $x$ & & \\
\hline \multicolumn{4}{|l|}{ Sicherheit } \\
\hline \multirow{4}{*}{$\begin{array}{l}\text { - Datenschutz bei Transaktionen } \\
\text { - Informationsverfügbarkeit } \\
\text { - Informationsvertraulichkeit } \\
\text { - Authentizität von Identitäten (Teilnehmer/- } \\
\text { innen) }\end{array}$} & $x$ & & \\
\hline & $x$ & & \\
\hline & $x$ & & \\
\hline & $x$ & & \\
\hline \multicolumn{4}{|l|}{ Verwaltung } \\
\hline \multirow{4}{*}{$\begin{array}{l}\text { - Integration von Dritten/Systeme } \\
\text { - Zugriffskontrolle } \\
\text {-Testbarkeit } \\
\text { - Systemflexibilität }\end{array}$} & $x$ & & \\
\hline & $x$ & & \\
\hline & $x$ & & \\
\hline & $x$ & & \\
\hline
\end{tabular}


weniger wichtig eingestuft. Große Aufmerksamkeit muss der Sicherheit und der Verwaltung geschenkt werden. Der Schutz der sensiblen Smart-Meter-Verbrauchsdaten, in Form einer spezifischen Verschlüsselung, muss bei der Umsetzung priorisiert werden. Dies spiegelten auch die Experteninterviews wider. Des Weiteren ist die Integration von weiteren Systemen und die Flexibilität der Blockchain ein wichtiger Erfolgsfaktor im Hinblick auf die Wechselprozesse und der Smart-Meter-Technologie.

\subsubsection{Regulatorische Betrachtung}

Die Experteninterviews haben einige Rahmenbedingungen und kritische Einschätzungen bezüglich des Anwendungsfalls aufgezeigt. Die Grundprinzipien der Energieunternehmen „Verursachergerecht“, „Energieeffizienz“ und „Förderung“ von Energieeinsparungen sind immer einzuhalten. Die Blockchain-Technologie darf außerdem zu keiner Einschränkung des Netzzuganges für die Endverbraucherinnen bzw. -verbraucher oder Prosumer führen. Das heißt, diese muss stets diskriminierungsfrei sein. Darüber hinaus muss die Verrechnung der Stromtarife auf Ist-Verbrauchsdaten basieren und mindestens einen Basistarif aufweisen. Die Abbildung von weiteren Wahl- oder dynamischen Tarifen ist unter der Bedingung einer schriftlichen, vertraglichen Regelung erlaubt. Dies ist darauf zurückzuführen, dass auch aufgrund der heutigen Messtechnik und des hohen administrativen Aufwands noch keine effiziente Abwicklung von Wahl- oder dynamischen Tarifen gewährleistet werden kann. Zudem bestehen begrenzte Anreize für solche Tarifmöglichkeiten, weil die Netzkosten weiterhin auf die einzelnen Endverbraucherinnen und -verbraucher abgewälzt werden können und das Fördern von netzdienlichem Verhalten zurzeit für den Verteilnetzbetreiber keinen größeren Mehrwert generiert. Allerdings wird vom Regulationscontroller hierbei erwähnt, dass es für die Kundin bzw. den Kunden einen Mehrwert schaffen könnte im Sinne einer Informationsplattform und als Werkzeug für Kosteneinsparungen. Dazu könnte es zu einer Prozessvereinfachung kommen, weil die Vergütung von Flexibilitäten, welche durch die Endverbraucher zur Verfügung gestellt werden, nun einfacher abgewickelt werden könnten. So könnte beispielsweise bei einem Kühlhaus festgelegt werden, daß es während zwei Stunden pro Tag keinen Strom beziehen darf und die Betreiber im Gegenzug eine entsprechende Vergütung erhalten werden. Eine weitere Vereinfachung könnte sich hierdurch bei der Abwicklung der Wechselprozesse ergeben, welche derzeitig aufwendig und kompliziert sind. Bei diesen Prozessen sind viele Stakeholder involviert, weshalb eine einheitliche Plattform einen großen Mehrwert schaffen könnte.

Generell ist dem Datenschutz große Aufmerksamkeit zu schenken. Bei den Verbrauchsdaten handelt es sich um sensible Daten, welche im Monopolbereich erfasst werden und nicht für marktdienliche Zwecke verwendet werden dürfen. Diese Daten dürften in einer solchen Plattform, auf welche mehrere Stakeholder Zugriff haben, nicht ohne weiteres gestreut werden. Auch Hein (2019) schenkt dem Datenschutz in ihrem Buch „Rechtliche Herausforderungen von Blockchain Anwendungen“" große Beachtung und gibt Folgendes zur Kenntnis: 
„Dazu bedarf es Regelungen, wie der Datenschutz in Blockchain-Netzwerken anzuwenden ist oder wie technische Implikationen, die die Umsetzung und Wahrung des Datenschutzes gewährleisten, grundsätzlich einzusetzen sind“ (Hein 2019, S. 32).

\subsubsection{Wirtschaftliche Betrachtung}

Mit einer blockchainbasierten Plattform könnte die Abwicklung von dynamischen Tarifen vereinfacht und effizient gestaltet werden. In dieser Hinsicht wäre eine feste Regelung möglich. Die Regeln könnten fälschungssicher in einem Smart Contract festgehalten werden und würden dadurch die Abwicklungskosten der Verrechnung minimieren. Dazu können mit dem Engpassmanagement Anreize geschaffen werden, zu welchem Zeitpunkt bzw. zu welcher Zeitperiode größere resp. kleinere Mengen an Energie bezogen wird, wobei sich die Anreize an den unterschiedlichen Tarifhöhen orientieren. So können durch ein effizienteres Netzengpassmanagement größere Netzausbauten verhindert werden. Zusätzlich spiegelt der Anwendungsfall „Blockchain for grid applications“ eine einheitliche Kollaborationsplattform für die über 600 VNB sowie den Marktakteur wider, was ebenfalls zu einer Vereinfachung des Informationsaustauschs und zu einer Reduktion des administrativen Aufwands führt. Die Plattform ermöglicht eine transparente Bereitstellung von Informationen, allerdings immer unter der Berücksichtigung des Datenschutzes. Basierend darauf ergibt sich die Möglichkeit, die Wechselprozesse effizienter zu gestalten, weil sich der Austausch über eine einheitliche Plattform mit fälschungssicheren Daten vereinfacht. Diesbezüglich könnte wiederum administrativer Aufwand eingespart werden. Allerdings erfordert der Anwendungsfall hohe und langfristige Investitionen in die ITInfrastruktur, weswegen eine detailliertere Überprüfung unverzichtbar ist.

\subsection{Kritische Betrachtung}

Mit der aktuellen Messtechnik sind die VNB noch nicht in der Lage, detailliertere Analysen bezüglich dem Verbrauchsverhalten durchzuführen. Dies ist mit dem Roll-out des Smart Meter verknüpft, welcher bis spätestens 2027 zu $80 \%$ abgeschlossen sein muss. Infolgedessen ist es zum gegenwärtigen Zeitpunkt schwierig einzuschätzen, ob es zukünftig überhaupt Sinn macht, dynamische Tarife einzusetzen und wie diese allenfalls ausgeprägt sein sollten. Aus diesem Grund ist ebenfalls schwer zu beurteilen, wie sinnvoll eine solche Tarifabbildung bzw. Verrechnung der Tarifkomponenten über die Blockchain überhaupt ist. Allerdings scheint die Blockchain als einheitliche und vertrauenswürdige Informationsaustauschplattform für die Verbrauchsdaten der Smart Meter und die damit verknüpften Wechselprozesse sinnvoll zu sein. Hierbei kann eine Effizienzsteigerung durch die Einsparung von administrativem Aufwand erreicht werden. Zudem wäre die Frage geklärt, wo die zukünftigen Verbrauchsdaten der Endverbraucherinnen und -verbraucher oder Prosumer gespeichert werden und wer über die Datenhoheit verfügen kann und darf. 
Die Blockchain würde hierbei der momentan durch den Gesetzgeber angedachte zentrale Datenhub ersetzten und könnte eine vertrauenswürdige sowie manipulationssichere Plattform schaffen.

Der Anwendungsfall „Blockchain for grid applications“ wurde zusammen mit Vertretern aus der Energiebranche erarbeitet. Mit dem Know-how der beiden Experten ist davon auszugehen, dass der Anwendungsfall einen praxisnahen Bezug aufweist. Des Weiteren wurde der Anwendungsfall in einer späteren Phase von insgesamt zwei Experten aus der Forschung und einem Experten aus dem Regulationsmanagement kritisch hinterfragt. Die Sicht des Netzbetreibers, sprich die der Personen, welche das Elektrizitätsnetz betreiben, müsste für einen allfälligen Einsatz der Blockchain ebenfalls miteinfließen und zusätzlich noch befragt werden. Auch die Sicht des Gesetzgebers sowie die der anderen Stakeholder müssten in einem weiteren Schritt noch eingeholt werden, um den Anwendungsfall auf die Praxistauglichkeit zu überprüfen. Generell zeigt die Arbeit die Grundlagen der BlockchainTechnologie auf und umschreibt die Risiken und Chancen im Hinblick auf den Anwendungsfall des VNB. Dabei wurde der kritischen Betrachtung große Aufmerksamkeit geschenkt.

\subsection{Fazit}

Der vorliegende Beitrag gibt einen Einblick in die Blockchain-Technologie resp. in deren Möglichkeiten und untersucht einen Anwendungsfall für den VNB. Der Anwendungsfall soll den Verrechnungsprozess, die Wechselprozesse sowie den Informationsaustausch von sämtlichen Marktakteure unterstützten. Im Hinblick auf den Datenschutz ist die Pseudonymität ein wichtiger Faktor, welcher unbedingt beachtet werden muss. Das heißt, die vertrauenskritischen Transaktionen können nicht direkt, sondern durch die Rückverfolgung von mehreren Transaktionen einem Individuum zugeteilt werden. Deswegen sollten hier differenzierte Verschlüsselungsmechanismen zur Anwendung kommen, damit die eindeutige Bestimmung einer Vertragspartei ausgeschlossen werden kann. Zusätzlich muss der Fokus auf eine konsortialen Blockchain mit dem Proof-of-Authority- oder Proof-of-Stake-Konsensmechanismus gelegt werden. Ein weiterer kritischer Faktor ist der Nutzen eines solchen Systems bzw. die Bereitschaft der Verteilnetzbetreiber, dass sie die Flexibilität und die Möglichkeit nutzen, anhand von dynamischen Tarifen das netzdienliche Verhalten zu fördern. Im Interview mit dem Experten aus dem Regulationsmanagement wurde dies ebenfalls als kritisch eingestuft, weil zurzeit noch keine Anreize seitens des Regulators vorhanden sind, um dies zu fördern. Allerdings ergäbe sich dadurch ein positiver Nutzen im Sinne einer Effizienzsteigerung der Tarifabwicklung. Auch der Informationsaustausch zwischen den einzelnen Marktakteuren könnte mit dem vorliegenden Anwendungsfall verbessert werden. Durch die einheitliche Plattform könnte sich ebenfalls eine Effizienzverbesserung der Wechselprozesse ergeben. Nebst der Prozessverbesserungen könnten die Vorteile der Blockchain genutzt werden; insbesondere die Dezentralität, die Transparenz und die Stabilität des Systems, allerdings stets unter der Berücksichtigung der Risiken wie beispielsweise die Unveränder- 
barkeit oder die Skalierbarkeit des Netzwerkes. Die technologische, regulatorische und wirtschaftliche Betrachtung zeigt auf, welche kritischen Punkte es im Allgemeinen zu beachten gilt und welche Einschränkungen momentan noch bestehen.

„Blockchain for grid applications“ ist eine zukunftsgerichtete Anwendung, welche für die Umsetzung weiter ausgearbeitet werden muss. Wie im vorherigen Kapitel beschrieben, ist der Fortschritt des Smart-Meter-Roll-outs maßgebend, damit detailliertere Analysen des Verbrauchsverhaltens durchgeführt werden können. Die Zusammenarbeit der Marktakteure spielt hierbei eine zentrale Rolle. Nur mit der Kooperation zwischen den unterschiedlichen Stakeholdern und der Bereitschaft der VNB, eine einheitliche Plattform zu bilden, könnte die Anwendung zukünftig einen Erfolg erzielen. Auch der Reifegrad und die kommende Entwicklung der Blockchain-Technologie ist umstritten und kann einen Einfluss auf den beschriebenen Anwendungsfall haben. An diesem Punkt ist auch zu erwähnen, dass sich die Blockchain-Technologie in einem dynamischen Umfeld bewegt, welches von stets neuen Ideen und Entwicklungen geprägt ist. Aufgrund dessen ist es schwierig zu beurteilen, welche Veränderungen die Technologie in der Zukunft noch durchläuft. In diesem Beitrag wurde der Fokus ausschließlich auf den VNB gesetzt, weshalb sich auch der Anwendungsfall nur auf die Anforderungen des VNB konzentriert. Allerdings ist es gut vorstellbar, dass weitere Bedürfnisse von anderen Bereichen und Stakeholdern miteinfließen könnten, wie beispielsweise die Abbildung der Stromprodukte für die Energielieferung. Hierfür könnten die EVU analog zum Netznutzungsentgelt ihre Produktkategorien in der Blockchain abbilden und anhand eines Smart Contracts automatisieren. Vorstellbar wären auch Modelle, bei welchen die Endverbraucherin bzw, der Endverbraucher ihren/seinen „Strommix“ selber zusammenstellt. Das heißt, er könnte den Mix aus erneuerbaren und nicht erneuerbaren Energien selbst bestimmten und auf sein Profil abstimmen. Dies würde dann bedeuten, dass nicht nur die Netznutzungskomponenten, sondern der gesamte Stromtarif (s. Abschn. 3.3.2) in der Blockchain abgebildet und über einen Smart Contract verrechnet wird.

Das Potenzial der Blockchain in der Energiewirtschaft wird hiermit noch einmal verdeutlicht. Vor allem im Hinblick auf das Datenmanagement und der Effizienzverbesserung von einzelnen Prozessen zeigt sie mit einer kritischen Betrachtung auf, welche Möglichkeiten und Anwendungen sich in der Zukunft ergeben könnten.

\section{Literatur}

Cathrin Hein, W. W. (2019). Rechtliche Herausforderungen von Blockchain-Anwendungen. Wiesbaden: Springer Fachmedien.

Daniel Höfelmann, P. S. (2019). Entscheidungshilfe für den Einsatz von Blockchain-Technologien in Unternehmen: Vier Frameworks im Vergleich. Frankfurt a. M.: Frankfurt School of FInance and Management. 
Eidgenössische Elektrizitätskomission ElCom. (2019). Fragen und Anworten zu neuartigen und dynamischen Netznutzugns- und Energieliefertarifen (27. Februar 2019). https://www.elcom.admin.ch/elcom/de/home/dokumentation/mitteilungen.html. Zugegriffen im April 2019.

Eidgenössische Elektrizitätskommision ElCom. (2020). Strompreis Webseite der ElCom. Strompreis Webseite der ElCom (05. Januar 2020). https://www.strompreis.elcom.admin.ch/Start.aspx. Zugegriffen im April 2019.

Peter, V. (2017). Blockchain in der Energiewirtschaft. Potenziale für Energieversorger. Berlin: BDEW Bundesverband der Energie- und Wasserwirtschaft.

Philip Richard, S. M. (2019). Blockchain in der integrierten Energiewende. dena-Multi-StakeholderStudie. Berlin: Deutsche Energie-Agentur GmbH.

Stefan Wunderlich, A. C. (2018). Energiemarkt mit Blockchain-Technolgie: Ein Marktmodell unter der Berücksichtigung bestehender Netzkomponenten und Marktakteure. Lüneburg: Carl von Ossietzky Universität.

swissgrid ag. (2012). Branchenempfehlung Strommarkt Schweiz. Netznutzungsmodell für das schweizerische Übertragungsnetz (25. Oktober 2012). https://www.strom.ch/de/download?keywords=NEtznutzungsmodell. Zugegriffen im April 2019.

VSE, Verband Schweizerischer Elektrizitätsunternehmen. (2013). VSE, Verband Schweizerischer Elektrizitätsunternehmen. (Oktober 2013). Anbindung von Regelpools an den Schweizer SDLMarkt. Von Branchenempfehlung Strommarkt Schweiz. https://www.strom.ch/de/download. Zugegriffen im April 2019.

VSE, Verband Schweizerische Elektrizitätsunternehmen. (2019). Mit welchem Netz in die Zukunft (19. November 2019). https://www.strom.ch/de/nachrichten/mit-welchem-netz-die-zukunft. Zugegriffen im April 2019.

VSE, Verband Schweizerischer Elektrizitätsunternehmen. (2018). Netznutzungsmodell für das schweizerische Verteilnetz. Branchenempfehlung Strommarkt Schweiz (5. Dezember 2018). https://www.strom.ch/de/download. Zugegriffen im April 2019.

Pascal Pfister Masterstudium in Betriebswirtschaft an der Berner Fachhochschule mit Vertiefung Corporate/Business Development. Langjährige Tätigkeit als Asset Manager der Infrastrukturanlagen bei Energie Wasser Bern, vor allem im Bereich der Wasser-, Gas- und Elektrizitätsversorgung. Aktuell beim VermögensZentrum in Bern als Associate Financial Consultant tätig.

Jan T. Frecè Tätigkeit in Lehre und Forschung am Departement Wirtschaft der Berner Fachhochschule, mit dem Themenschwerpunkten Nachhaltigkeit im digitalen Raum, nachhaltiges Unternehmertum, funktionale Unternehmenswerte sowie Umgang mit Daten in einer digitalisierten Welt. Zuvor tätig im Sustainability Consulting und als IT Solution Architect im internationalen IT-Sektor. 
Open Access Dieses Kapitel wird unter der Creative Commons Namensnennung 4.0 International Lizenz (http://creativecommons.org/licenses/by/4.0/deed.de) veröffentlicht, welche die Nutzung, Vervielfältigung, Bearbeitung, Verbreitung und Wiedergabe in jeglichem Medium und Format erlaubt, sofern Sie den/die ursprünglichen Autor(en) und die Quelle ordnungsgemäß nennen, einen Link zur Creative Commons Lizenz beifügen und angeben, ob Änderungen vorgenommen wurden.

Die in diesem Kapitel enthaltenen Bilder und sonstiges Drittmaterial unterliegen ebenfalls der genannten Creative Commons Lizenz, sofern sich aus der Abbildungslegende nichts anderes ergibt. Sofern das betreffende Material nicht unter der genannten Creative Commons Lizenz steht und die betreffende Handlung nicht nach gesetzlichen Vorschriften erlaubt ist, ist für die oben aufgeführten Weiterverwendungen des Materials die Einwilligung des jeweiligen Rechteinhabers einzuholen.

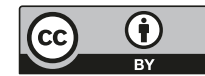

